\title{
CONTINUOUS ACTIONS OF COMPACT LIE GROUPS ON RIEMANNIAN MANIFOLDS
}

\author{
DAVID HOFFMAN AND L. N. MANN 1
}

\begin{abstract}
M. H. A. Newman proved that if $M$ is a connected topological manifold with metric $d$, there exists a number $\varepsilon>0$, depending only upon $M$ and $d$, such that every compact Lie group acting effectively on $M$ has at least one orbit of diameter at least $\varepsilon$. In this paper the authors consider the case where $M$ is a Riemannian manifold and $d$ is the distance function on $M$ arising from the Riemannian metric. They obtain estimates for $\varepsilon$ in terms of convexity and curvature invariants of $M$.
\end{abstract}

1. Introduction. In 1931 M. H. A. Newman proved the following result.

THEOREM (NEWMAN [8]). If $M$ is a connected topological manifold with metric $d$, there exists a number $\varepsilon=\varepsilon(M, d)>0$, depending only upon $M$ and $d$, such that every compact Lie group $G$ acting effectively on $M$ has at least one orbit of diameter at least $\varepsilon$.

Recently several investigators, including ourselves [4], [6], [7], have studied compact groups of isometries on a Riemannian manifold $M$ and have obtained estimates for $\varepsilon$ in terms of convexity and curvature invariants of $M$. In this paper we consider continuous actions of compact Lie groups on a Riemannian manifold $M$ and we obtain results which compare quite favorably with the results for isometric actions. Moreover, our arguments are surprisingly simple. Specifically, we have obtained the following results: We call a subset $S$ of $M$ convex if for every pair of points in $S$ there exists a unique distance measuring geodesic in $S$ joining them. For $x \in M$, the radius of convexity of $M$ at $x$, which we denote by $r_{x}$, is defined as the supremum of the radii of all convex embedded open balls centered at $x$.

THEOREM 1. Let $M$ be a Riemannian manifold with nonpositive sectional curvature. Let

$$
\bar{r}=\sup _{x \in M} r_{x}
$$

and suppose $r$ is any number, $0<r<\bar{r}$. If $G$ is any compact Lie group acting

Received by the editors March 24, 1976.

AMS (MOS) subject classifications (1970). Primary 57E10, 53C20; Secondary 54H15.

Key words and phrases. Newman's theorem on periodic transformations, diameter of orbits, radius of convexity.

${ }^{1}$ The authors were partially supported by grants from the National Science Foundation. 
continuously and effectively on $M$, then there exists at least one orbit of diameter at least $r / 2$. In particular if $\bar{r}=+\infty$, there exist orbits of arbitrarily large diameters.

The technique of proof depends upon the original approach of Newman as presented in a paper by Andreas Dress [2] together with the well-known fact that the exponential map locally stretches distances for manifolds of nonpositive curvature. For manifolds of bounded curvature $K \leqslant b^{2}$, we obtain an analogous result by using a technique similar to that of Hoffman [4] which compares distances locally on $M$ with distances on a space form of constant positive curvature $b^{2}$.

THEOREM 2. Let $M$ be a Riemannian manifold with curvature bounded above by a positive constant $b^{2}$. Suppose $r$ is any number, $0<r<\min \left\{\pi / 2 \cdot b^{-1}, \bar{r}\right\}$. If $G$ is any compact Lie group acting continuously and effectively on $M$, then there exists at least one orbit of diameter at least $2 r /(\pi+2)$.

As for a best possible type result, we have the following candidate.

THEOREM 3. Let $G$ be a compact Lie group acting continuously and effectively on a compact orientable Riemannian manifold $M$. Then there exists at least one orbit which is not contained in any open convex subset of $M$.

In [9], P. A. Smith gave a generalization of Newman's Theorem to cohomology manifolds. In this paper Smith claims [9, p. 448] that if a compact Lie group acts continuously and effectively on an $n$-sphere $S^{n}$ (with the standard metric) there exists at least one orbit which is not contained in any open hemisphere. Theorem 3 is a natural generalization of Smith's claim. The existence of such a result was suggested by the techniques of G. Bredon in [1, III.9] where he gives a proof of Smith's version of the Newman Theorem. The assumptions of compactness and orientability appear to be needed only for technical reasons.

2. Proof of Theorems 1 and 2. We will use the following two results:

A. Lemma (A. Dress [2]). Let $U$ be an open, relatively compact and connected subset of $R^{n}$. If $Z_{p}$ acts continuously, effectively and invariantly on $\bar{U}$, then

$$
\begin{aligned}
D & =\operatorname{Max}\{\operatorname{Min}\{\|x-y\| \mid y \in \partial \bar{U}\} \mid x \in U\} \\
& \leqslant C=\left\{\operatorname{Max}\|x-\tau x\| \mid \tau \in Z_{p}, x \in \partial \bar{U}\right\} .
\end{aligned}
$$

Here $\|x-y\|$ is the euclidean norm in $R^{n}$.

B. Proposition. Suppose $K \leqslant b^{2}$ (respectively $K \leqslant 0$ ) on a Riemannian manifold $M$ with distance function $d$. Let $B_{r}(z)=\{y \mid d(y, z)<r\}$ be a convex embedded ball centered at $z$ in $M$. Suppose further that $r<\pi b^{-1} / 2$ (respectively $0<r<\infty$ when $K \leqslant 0)$. For any $x, y \in B_{r}(z)$, if $\hat{x}=\exp _{z}^{-1} x$ and $\hat{y}$ $=\exp _{z}^{-1} y$, then $d(x, y) \geqslant(2 / \pi)\|\hat{x}-\hat{y}\|$ (respectively $d(x, y) \geqslant\|\hat{x}-\hat{y}\|$ when $K \leqslant 0)$. Here $\|x-y\|$ is the euclidean norm in the tangent space $M_{z}$. 
Lemma $\mathrm{A}$ appears as Lemma 3 in [2]. Proposition $\mathrm{B}$, for the case $K \leqslant 0$, is a well-known fact. The proof of Proposition B for Riemannian manifolds with curvature bounded above by a positive constant will be presented in the next section.

We prove Theorems 1 and 2 simultaneously. Without loss of generality, we may assume $G=Z_{p}$. Fix any $z \in M$ and let $r_{z}=$ the radius of convexity at $z$. For any $r>0$ satisfying

$$
r< \begin{cases}r_{z} & \text { if } K \leqslant 0 \\ \operatorname{Min}\left\{r_{z}, \pi / 2 \cdot b^{-1}\right\} & \text { if } K \leqslant b^{2}\end{cases}
$$

and any $\alpha, 0<\alpha<1$, suppose that

$$
d(x, \tau x)<(1-\alpha) r \text { for all } x \in M, \text { all } \tau \in Z_{p} .
$$

Define $U=\cup_{\tau \in Z_{p}} \tau B_{\alpha r}(z)$. By construction, $\bar{U}$ is $Z_{p}$-invariant. Furthermore, by $(\mathrm{H}), \bar{B}_{\alpha r}(z) \subset \bar{U} \subset \bar{B}_{r}(z)$.

Now lift the action of $Z_{p}$ on $\bar{U}$ to an action of $Z_{p}$ on the closed set $\exp _{z}^{-1} \bar{U}$, i.e. let $\tau \in Z_{p}$ act on $\exp _{z}^{-1} \bar{U}$ by $\exp _{z}^{-1} \circ \tau \circ \exp _{z}$. For convenience, we will let $U_{\Lambda}=\exp _{z}^{-1} U$. Clearly $\bar{U}_{\Lambda}$ is $Z_{p}$ invariant and

$$
\begin{aligned}
\left\{\hat{x} \in M_{z} \mid\|\hat{x}\| \leqslant \alpha r\right\} & =\exp _{z}^{-1} \bar{B}_{\alpha r}(z) \subset \bar{U}_{\Lambda} \\
& \subset \exp _{z}^{-1} \bar{B}_{r}(z)=\left\{\hat{x} \in M_{z} \mid\|\hat{x}\| \leqslant r\right\} .
\end{aligned}
$$

The left-hand inclusion implies

$$
D=\operatorname{Max}\left\{\operatorname{Min}\left\{\|\hat{x}-\hat{y}\| \mid \hat{y} \in \partial \bar{U}_{\wedge}\right\} \mid \hat{x} \in U_{\wedge}\right\} \geqslant \alpha r .
$$

(Simply let $\hat{x}=0$.) Since $\bar{B}_{r}(z)$ is a convex, embedded ball with $r<\pi / 2 \cdot b^{-1}$ when $K \leqslant b^{2}(r<\infty$ when $K \leqslant 0)$, we may apply Proposition B, which, together with $(\mathrm{H})$ implies

$$
C=\operatorname{Max}\left\{\|\hat{x}-\tau \hat{x}\| \mid \hat{x} \in \partial \bar{U}_{\wedge}, \tau \in Z_{p}\right\}< \begin{cases}(1-\alpha) r & \text { if } K \leqslant 0 \\ (1-\alpha) \pi r / 2 & \text { if } K \leqslant b^{2}\end{cases}
$$

But, by Lemma $A, D \leqslant C$. This implies

$$
\alpha r< \begin{cases}(1-\alpha) r & \text { if } K \leqslant 0 \\ (1-\alpha) \pi r / 2 & \text { if } K \leqslant b^{2}\end{cases}
$$

or

$$
\alpha< \begin{cases}1 / 2 & \text { if } K \leqslant 0 \\ \pi / \pi+2 & \text { if } K \leqslant b^{2}\end{cases}
$$

Therefore, $(\mathrm{H})$ is false for 


$$
\alpha= \begin{cases}1 / 2 & \text { if } K \leqslant 0 \\ \pi / \pi+2 & \text { if } K \leqslant b^{2}\end{cases}
$$

i.e. there exists an $x \in M$ whose orbit has diameter at least $r / 2$ if $K \leqslant 0$; $2 r / \pi+2$ if $K \leqslant b^{2}$. This completes the proof of Theorems 1 and 2 .

REMARK. For $Z_{2}$ actions, Lemma $A$ may be strengthened to say that $2 D \leqslant C$ (there is a misprint on p. 206 of [2]). Moreover if $G$ is not a finite group of odd order, it must contain an involution. Using these facts in the above argument shows that:

COROLlary. Let $M$ be a Riemannian manifold with sectional curvature $K \leqslant b^{2}$ (respectively $K \leqslant 0$ ). Suppose $r$ satisfies $0<r<\operatorname{Min}\left\{\pi / 2 \cdot b^{-1}, \bar{r}\right\}$ (respectively $0<r<\bar{r}$ when $K \leqslant 0$ ). If $G$ is a compact Lie group acting continuously and effectively on $M$ and if $G$ is not a finite group of odd order, then there exists at least one orbit of diameter at least $4 r / \pi+4$ (respectively $2 r / 3$ when $K \leqslant 0)$.

3. A distance preserving property of the exponential map. In this section we outline a proof of Proposition B in the case where the curvature of $M$ is bounded above by a positive constant $b^{2}$.

Let $z \in M$ and choose any $r, 0<r<\operatorname{Min}\left(r_{z}, \pi / 2 \cdot b^{-1}\right)$. For any $x, y$ $\in B_{r}(z)$, we must show $d(x, y) \geqslant(2 / \pi)\|\hat{x}-\hat{y}\|$.

We proceed as follows. Let $S^{n}\left(b^{-1}\right)$ be the $n$-sphere of constant curvature $b^{2}$, and fix $p \in S^{n}\left(b^{-1}\right)$.

The choice of $r$ ensures that $B_{r}(z)$ and $\tilde{B}_{r}(p)$, the geodesic ball of radius $r$ centered at $p \in S^{n}\left(b^{-1}\right)$, are both convex. Identify $M_{z}$ with $S^{n}\left(b^{-1}\right)_{p}$ by a linear isometry $i$. We have the bijection

$$
B_{r}(z) \stackrel{\exp _{z}^{-1}}{\longrightarrow} M_{z} \stackrel{i}{\rightarrow} S^{n}\left(b^{-1}\right)_{p} \stackrel{\widetilde{\exp _{p}}}{\longrightarrow} \tilde{B}_{r}(p) .
$$

Here, $\widetilde{\exp }_{p}$ is the exponential map of $S^{n}\left(b^{-1}\right)$ restricted to the tangent space at $p$. Let $\eta=\widetilde{\exp }_{p} \circ i \circ \exp _{z}^{-1}$.

Since $x, y \in B_{r}(z)$, neither $x$ nor $y$ is conjugate to $z$ along any geodesic in $B_{r}(z)$. Similarly, $\eta x, \eta y \in \tilde{B}_{r}(p)$ are not conjugate along any geodesic in $\tilde{B}_{r}(p)$. Therefore we may apply the Rauch Comparison Theorem $[5$, p. 76] to conclude:

If $\gamma$ is a length-measuring geodesic connecting $x$ to $y$ in $B_{r}(z)$,

$$
\tilde{d}(\eta x, \eta y) \leqslant \text { Length }(\eta \circ \gamma) \leqslant \text { Length }(\gamma)=d(x, y) .
$$

Here $\tilde{d}$ is the distance function in $S^{n}\left(b^{-1}\right)$. Now suppose $\delta$ is a lengthmeasuring geodesic joining $\eta x$ to $\eta y$ in $\tilde{B}_{r}(p) \subset S^{n}\left(b^{-1}\right)$. The curve $\widetilde{\exp }_{p}^{-1} \circ \delta$ connects $\exp _{p}^{-1} \eta x$ to $\widetilde{\exp }_{p}^{-1} \eta y$, so

$$
\left\|\widetilde{\exp }_{p}^{-1} \eta x-\widetilde{\exp }_{p}^{-1} \eta y\right\| \leqslant \text { Length }\left(\widetilde{\exp }_{p}^{-1} \circ \delta\right) .
$$

Here length is measured in $S^{n}\left(b^{-1}\right)_{p}$. Since $i$ is an isometry (and $i \hat{x}$ 
$=\widetilde{\exp }_{p}^{-1} \eta x$, etc. $)$

$$
\|x-y\| \leqslant \text { Length }\left(\widetilde{\exp }_{p}^{-1} \circ \delta\right) .
$$

We now wish to compare the length of $\widetilde{\exp }_{p}^{-1} \circ \delta$ with the length of $\delta \subset S^{n}\left(b^{-1}\right)$. A straightforward computation, using polar coordinates to express $\widetilde{\exp }_{p}$ and the arc-length formula, yields

$$
((\sin b r) / b r) \text { Length }\left(\exp ^{-1} \circ \delta\right) \leqslant \text { Length } \delta \text {. }
$$

Since $r<\pi / 2 \cdot b^{-1}$ and $\delta$ measures length,

$$
2 / \pi \cdot \text { Length }\left(\exp _{p}^{-1} \delta\right) \leqslant \tilde{d}(\eta x, \eta y)
$$

Combining (1), (2) and (3),

$$
2\|\hat{x}-\hat{y}\| / \pi \leqslant d(x, y),
$$

which is the desired inequality.

4. Proof of Theorem 3. In [1, pp. 154-156] Bredon proves a version of Newman's Theorem due to P. A. Smith. As a preliminary he gives a result, Theorem 9.3 of [1], where he proves:

If $M$ is a compact orientable manifold and $\mathcal{U}$ is any open covering of $M$ such that $H^{n}(K(\mathcal{Q}), Z) \rightarrow \check{H}^{n}(M, Z)$ is onto, then there does not exist a continuous effective action of a compact Lie group on $M$ such that each orbit is contained in some member of थ.

Here, $H(K(\mathcal{Q}), Z)$ is the integral cohomology of $K(\mathcal{Q})$, the nerve of the covering $\mathscr{Q}$, and $\check{H}(M, Z)$ is the integral Čech cohomology. The map is the natural induced map.

So now let $\mathcal{Q}$ be the covering consisting of all open, convex sets of the Riemannian manifold $M$.

Since the intersection of convex sets is a convex set and convex sets are contractible, $H^{q}(|\sigma|, Z)=0$ for all $\sigma \in K(\mathcal{Q}), q \geqslant 1$. By Leray's Theorem [3, p. 44], the induced map $H^{q}(K($ Q $), Z) \rightarrow \check{H}^{q}(M, Z)$ is an isomorphism for all $q \geqslant 0$. In particular, $H^{n}(K($ Q $), Z) \rightarrow \check{H}^{n}(M, Z)$ is onto. Thus, Bredon's result is applicable to the covering by convex sets, proving Theorem 3 .

\section{REFERENCES}

1. Glen E. Bredon, Introduction to compact transformation groups, Academic Press, New York, 1972.

2. Andreas Dress, Newman's theorems on transformation groups, Topology 8 (1969), 203-207. MR 38 \#6629.

3. R. C. Gunning, Lectures on Riemann surfaces, Princeton Univ. Press, Princeton, N.J., 1966. MR 34 \# 7789.

4. David Hoffman, The diameter of orbits of compact groups of isometries; Newman's theorem for non-compact manifolds, Trans. Amer. Math. Soc. (to appear).

5. S. Kobayashi and K. Nomizu, Foundations of differential geometry, Vol. II, Interscience, New York, 1969. MR 38 \#6501. 
6. M. C. Ku, Newman's theorem for compact Riemannian manifolds, University of Massachusetts (preprint).

7. L. N. Mann and J. L. Sicks, Newman's theorem in the Riemannian category, Trans. Amer. Math. Soc. 210 (1975), 259-266.

8. M. H. A. Newman, A theorem on periodic transformations of spaces, Quart. J. Math. 2 (1931), $1-9$.

9. P. A. Smith, Transformations of finite period. III: Newman's theorem, Ann. of Math. (2) 42 (1941), 446-458. MR 2, 324.

Department of Mathematics, University of Massachusetts, Amherst, Massachusetts 01002 